\title{
INJECTIVITY, QUASICONFORMAL REFLECTIONS AND THE LOGARITHMIC DERIVATIVE
}

\author{
REUVEN HARMELIN
}

\section{Introduction}

Let $C$ be a $K$-quasicircle in the complete complex plane $\hat{\mathbf{C}}=\mathbf{C} \cup\{\infty\}$ (i.e., $C$ is the image of the real axis $\hat{\mathbf{R}}$ under a $K$-quasiconformal automorphism of $\hat{\mathbf{C}}$, with $K \geqq 1$ ), and $D_{1}, D_{2}$ to be the components of $\hat{\mathbf{C}} \backslash C$, such that $\infty \in \bar{D}_{2}=D_{2} \cup C$. Let $\varrho_{i}(z)|d z|$ be the Poincaré metric in $D_{i}, i=1,2$, with curvature -4. In [2] Ahlfors proved the following injectivity criterion in terms of the Schwarzian derivative

$$
S_{f}(z)=\left[\left(f^{\prime \prime} \mid f^{\prime}\right)^{\prime}-\frac{1}{2}\left(f^{\prime \prime} \mid f^{\prime}\right)^{2}\right](z), \quad z \in D_{1} .
$$

The or em A. If $C$ is a K-quasicircle, then there exists a positive constant $\varepsilon=\varepsilon(K)$, depending only on $K$, such that $f$ is univalent in $D_{1}$ whenever it is meromorphic there, with a nonvanishing derivative and

$$
\left\|S_{f}\right\|_{2, D_{1}}=\sup _{z \in D_{1}} \varrho_{1}(z)^{-2}\left|S_{f}(z)\right| \leqq \varepsilon .
$$

Moreover, strict inequality in (1.1) implies the existence of a quasiconformal extension of $f$ to $\hat{\mathbf{C}}$.

Writing

$$
S_{f}=T_{f}^{\prime}-\frac{1}{2} T_{f}^{2}, \quad T_{f}=f^{\prime \prime} \mid f^{\prime}
$$

and applying Lemma 3 of [9], one can easily show that the correspondence $\pi: T_{f} \rightarrow S_{f}$ is continuous with respect to the norms $\left\|S_{f}\right\|_{2, D_{1}}$, defined in (1.1), and

$$
\left\|T_{f}\right\|_{1, D_{1}}=\sup _{z \in D_{1}} \varrho_{1}(z)^{-1}\left|T_{f}(z)\right| .
$$

This observation yields the following analogue for Theorem A.

Theorem B. If $C$ is a $K$-quasicircle, then there exists a positive constant $\varepsilon_{1}=$ $\varepsilon_{1}(K)$, depending only on $K$, such that every analytic function $f$ in $D_{1}$, with $f^{\prime} \neq 0$, is univalent whenever

$$
\left\|T_{f}\right\|_{1, D_{1}} \leqq \varepsilon_{1},
$$

and if there is a strict inequality in (1.3), $f$ has a quasiconformal extension to $\hat{C}$. 
Remark. Martio and Sarvas [11] proved the injectivity part of Theorems A and $\mathrm{B}$, using the uniformity property of quasidiscs. On the other hand, Gehring [8] and Astala-Gehring [5] proved that these injectivity criteria are valid only on quasidiscs.

In this paper, a different quantitative proof for Theorem B is provided, based on Ahlfors' idea of the proof of Theorem A but utilizing a slight modification of his quasiconformal extension formula. First we show that two properties of quasiconformal reflections, which have been proven in [2] under the assumption $\infty \in C$, remain valid also if $\infty \in \bar{D}_{2}$. Then Theorem $\mathrm{B}$ is proved and in the last section it is applied to the universal Teichmüller space.

\section{Quasiconformal reflections}

An automorphism $h$ of $\mathrm{C}$ is a $K$-quasiconformal reflection at $C$ if

(i) $\left.h\right|_{C}=$ id and $h \circ h=$ id on $\hat{\mathbf{C}}$.

(ii) The mapping $z \rightarrow \overline{h(z)}$ is a (sense-preserving) $K$-quasiconformal mapping, i.e.,

$$
\left|\frac{\partial h}{\partial z}\right| \leqq \frac{K-1}{K+1}\left|\frac{\partial h}{\partial \bar{z}}\right|<\left|\frac{\partial h}{\partial \bar{z}}\right| .
$$

Properties (i) and (ii) yield a third one:

(iii) $h\left(D_{i}\right)=D_{j}, \quad i \neq j, \quad i, j=1,2$.

Lemma 1 (Ahlfors [2]). If $\mathbf{C}$ is a $K$-quasicircle, $\mathbf{C}$ admits a $K^{2}$-quasiconformal reflection.

Lemma 2 (cf [2]). Let $h: \hat{\mathbf{C}} \rightarrow \hat{\mathrm{C}}$ be a K-quasiconformal reflection at $C$. If $\infty \in \bar{D}_{2}$ and $z_{0} \in C$, then

$$
\left|h(z)-z_{0}\right| \leqq \lambda(K)\left|z-z_{0}\right|, \text { for all } z \in D_{2},
$$

where

$$
\lambda(K)=\left[\mu^{-1}\left(\frac{\pi K}{2}\right)\right]^{-2}-1
$$

and $\mu(r)$ is the conformal module of the Grötzsch extremal ring domain separating the exterior of the unit disc $B$ from the interval $(0, r)$ for $0 \leqq r<1$ (see [10], pp. $53,81)$.

Proof. Let $f$ be a conformal mapping of the upper half plane $U$ onto $D_{2}$, with $f(\infty)=\infty$ if $\infty \in C$, or $f(\infty)=z_{0} \in C$ if $\infty \in D_{2}$. Define

$$
\omega(\zeta)=\left\{\begin{array}{lll}
f(\zeta) & \text { for } \quad \zeta \in U, \\
h(f(\bar{\zeta})) & \text { for } \quad \zeta \in L=\mathbf{C} \bar{U} .
\end{array}\right.
$$


Then $C=\omega(\mathbf{R})$ and $\omega: \hat{\mathbf{C}} \rightarrow \mathbf{C}$ is $K$-quasiconformal. Thus, if $z \in D_{2}$, then

and

$$
z=\omega(\zeta)=f(\zeta) \text { for some } \zeta \in U
$$

$$
h(z)=h(f(\zeta))=\omega(\bar{\zeta}), \quad \bar{\zeta} \in L .
$$

First, if $\infty \in C$, take $\zeta_{0}=\omega^{-1}\left(z_{0}\right) \in \mathbf{R}$. Then the cross-ratio

$$
\left|\left(\zeta, \zeta, \zeta_{0}, \infty\right)\right|=\left|\frac{\bar{\zeta}-\zeta_{0}}{\zeta-\zeta_{0}}\right|=1,
$$

and (2.1) follows by the quasi-invariance of the cross-ratio under quasiconformal mappings (see [1]), and since $\omega$ maps $\left\{\bar{\zeta}, \zeta, \zeta_{0}, \infty\right\}$ onto $\left\{h(z), z, z_{0}, \infty\right\}$, respectively.

Next, if $\infty \in D_{2}$, let $\omega^{-1}(\infty)=x \in U$. Then

$$
|(\zeta, \zeta, \infty, \alpha)|=\left|\frac{\zeta-\alpha}{\zeta-\alpha}\right|<1 \text { for } \zeta \in U,
$$

and (2.1) is obtained again since $\omega:\{\zeta, \zeta, \infty, \alpha\} \rightarrow\left\{h(z), z, z_{0}, \infty\right\}$, respectively.

Q.e.d.

Lemma 3 (cf. [2]). If $C$ admits a $K$-quasiconformal reflection, then there exists a $\mathscr{K}$-quasiconformal reflection $H$ at $C$ satisfying

$$
\varrho_{j}(H(z))|d H(z)| \leqq \mathscr{L} \cdot \varrho_{i}(z)|d z|, \quad z \in D_{i}, \quad i \neq j, \quad i, j=1,2
$$

for some constants $\mathscr{K}=\mathscr{K}(K)$ and $\mathscr{L}=\mathscr{L}(K)$ depending only on $K$.

The proof of Lemma 3 is given in [2]. The assumption $\infty \in C$ in it might be dropped, since it was needed only for deriving the corresponding inequality in the Euclidean metric. From Ahlfors' proof in [2] and the estimates in [3] and [7] one gets the following estimates:

$$
\mathscr{K}(K) \leqq M^{2}, \quad \mathscr{L}(K) \leqq 4 M^{2}(M+1), \text { where } \quad M=\lambda(K) .
$$

\section{Proof of Theorem B}

Assume first that $f$ has the following property:

(*) $f$ is locally conformal on some domain containing $\bar{D}_{1}=D_{1} \cup C$.

By Lemma 1, $C$ admits a $K^{2}$-quasiconformal reflection, and hence, by Lemma 3 , there exists another $\mathscr{K}$-quasiconformal reflection $H$ at $C$, with $\mathscr{K} \leqq M^{2}=\lambda\left(K^{2}\right)^{2}$, which satisfies Condition (2.2) with $\mathscr{L} \leqq 4 M^{2}(M+1)$.

Now, extend $f$ into $D_{2}$ as follows:

$$
\tilde{f}(\zeta)=f(z)+(\zeta-z) f^{\prime}(z), \quad \zeta \in D_{2}, \quad z=H(\zeta) \in D_{1} .
$$


This extension has the complex derivatives

$$
\frac{\partial \tilde{f}}{\partial \bar{\zeta}}=(\zeta-z) \frac{\partial z}{\partial \bar{\zeta}} f^{\prime \prime}(z) \quad \text { and } \quad \frac{\partial \tilde{f}}{\partial \zeta}=f^{\prime}(z)+(\zeta-z) \frac{\partial z}{\partial \zeta} f^{\prime \prime}(z), \quad z=H(\zeta) \in D_{1},
$$

and hence it has the complex dilatation

$$
\tilde{\mu}(\zeta)=\frac{(\zeta-z) \frac{\partial z}{\partial \zeta} T_{f}(z)}{1+(\zeta-z) \frac{\partial z}{\partial \zeta} T_{f}(z)}, \quad \zeta \in D_{2}, \quad z=H(\zeta) \in D_{1} .
$$

But by Lemma 2 we have for all $\zeta_{0} \in C$

$$
|\zeta-H(\zeta)| \leqq\left|\zeta-\zeta_{0}\right|+\left|\zeta_{0}-H(\zeta)\right| \leqq(1+\lambda(\mathscr{K}))\left|\zeta-\zeta_{0}\right|, \quad \zeta \in D_{2} .
$$

Choose $\zeta_{0} \in C$ such that $\left|\zeta-\zeta_{0}\right|=d(\zeta, C)$ and apply the inequality $d(\zeta, C) \leqq \varrho_{2}(\zeta)^{-1}$, $\zeta \in D_{2}$ (by Schwarz' lemma). Thus

$$
|\zeta-H(\zeta)| \leqq(1+\lambda(\mathscr{K})) \varrho_{2}(\zeta)^{-1}, \quad \zeta \in D_{2} .
$$

On the other hand, Property (2.2) of $H$ implies

$$
\left|\frac{\partial H(\zeta)}{\partial \bar{\zeta}}\right| \leqq \mathscr{L} \cdot \varrho_{2}(\zeta) / \varrho_{1}(H(\zeta)), \quad \zeta \in D_{2},
$$

and since $\zeta \rightarrow \overline{H(\zeta)}$ is $\mathscr{K}$-quasiconformal, it follows that

$$
\left|\frac{\partial H(\zeta)}{\partial \zeta}\right| \leqq q\left|\frac{\partial H(\zeta)}{\partial \zeta}\right| \text { with } \quad q=q(K)=\frac{\mathscr{K}-1}{\mathscr{K}+1}<1 .
$$

From (3.2), (3.3'), (3.4) and (3.5) we conclude

$$
|\tilde{\mu}(\zeta)| \leqq \frac{A(K) \varrho_{1}(z)^{-1}\left|T_{f}(z)\right|}{1-q(K) A(K) \varrho_{1}(z)^{-1}\left|T_{f}(z)\right|}, \quad \zeta \in D_{2}, \quad z=H(\zeta) \in D_{1},
$$

where $A(K)=4 M^{2}(M+1)\left(1+\lambda\left(M^{2}\right)\right), q(K)=\left(M^{2}-1\right) /\left(M^{2}+1\right), M=\lambda\left(K^{2}\right)$. Thus $\|\tilde{\mu}\|_{\infty} \leqq k<1$ whenever $f$ satisfies

$$
\left\|T_{f}\right\|_{1, D_{1}} \leqq A(K)^{-1} \frac{k}{1+q(K) k}<\varepsilon_{1}=\frac{A(K)^{-1}}{1+q(K)}=\varepsilon_{1}(K) .
$$

Hence, the mapping

$$
F(z)=\left\{\begin{array}{lll}
f(z) & \text { for } & z \in \bar{D}_{1}=D_{1} \cup C, \\
\tilde{f}(z) & \text { for } & z \in D_{2}
\end{array}\right.
$$

is a local homeomorphism all over $\hat{\mathbf{C}}$, and therefore it is an automorphism of $\hat{\mathbf{C}}$, thus proving the theorem whenever $f$ satisfies (*). 
Now, if $f$ does not satisfy $(*)$, let $\left\{G_{n}\right\}$ be any decreasing sequence $-\bar{G}_{n+1} \subset G_{n}-$ of simply-connected hyperbolic domains, such that $D_{1}=\bigcap_{n=1}^{\infty} G_{n}$. Let $g_{n}: G_{n} \rightarrow D_{1}$ be the conformal mapping with $g_{n}\left(z_{0}\right)=z_{0}$ and $g_{n}^{\prime}\left(z_{0}\right)>0$ for some fixed $z_{0} \in D_{1} \subset G_{n}$. Then $\left\{g_{n}\right\}$ converges to the identity mapping locally uniformly on $D_{1}$. Define

$$
\psi_{n}=\left(T_{f} \circ g_{n}\right) g_{n}^{\prime}, \quad n \geqq 1 .
$$

Then $\left\{\psi_{n}\right\}$ converges to $T_{f}$ locally uniformly on $D_{1}$. Note that each $\psi_{n}$ is analytic on $\bar{D}_{1} \subset G_{n}$, and by

$$
\varrho_{G_{n}}(z) \leqq \varrho_{1}(z), \quad z \in D_{1} \subset G_{n}
$$

(which is a consequence of Schwarz' lemma) it follows that

$$
\begin{aligned}
& \varrho_{1}(z)^{-1}\left|\psi_{n}(z)\right| \leqq \varrho_{G_{n}}(z)^{-1}\left|g_{n}^{\prime}(z)\right|\left|T_{f}\left(g_{n}(z)\right)\right| \\
& =\varrho_{1}\left(g_{n}(z)\right)^{-1}\left|T_{f}\left(g_{n}(z)\right)\right| \leqq\left\|T_{f}\right\|_{1, D_{1}}, \quad z \in D_{1} .
\end{aligned}
$$

Thus if $f_{n}$ is any solution of the equation

$$
y^{\prime \prime}-\psi_{n} y^{\prime}=0 \text { in } G_{n},
$$

then $f_{n}$ satisfies property (*) in addition to the assumptions of the theorem and $T_{f_{n}}=\psi_{n}$. Furthermore, since the general solution of (3.9) is of the form $a f_{n}+b$, we can choose the solution $f_{n}$ that fixes two given points on $C$. Hence, the sequence $\left\{F_{n}\right\}$ of mappings defined by

$$
F_{n}(\zeta)= \begin{cases}f_{n}(\zeta) & \text { for } \zeta \in \bar{D}_{1}, \\ f_{n}(z)+(\zeta-z) f_{n}^{\prime}(z) & \text { for } \zeta \in D_{2}, \quad z=H(\zeta) \in D_{1}\end{cases}
$$

form a normal family of quasiconformal automorphisms of $\hat{C}$. We conclude that $\left\{F_{n}\right\}$ contains a subsequence converging locally uniformly on $\hat{\mathbf{C}}$ to

$$
F_{0}(\zeta)=\left\{\begin{array}{lll}
f_{0}(\zeta) & \text { for } & \zeta \in \bar{D}_{1} \\
\tilde{f}_{0}(\zeta) & \text { for } & \zeta \in D_{2}
\end{array}\right.
$$

where $f_{0}=\lim _{n \rightarrow \infty} f_{n}$ is conformal and univalent in $D_{1}$, with $T_{f_{0}}=\lim _{n \rightarrow \infty} T_{f_{n}}=$ $T_{f}$, and

$$
\tilde{f}_{0}(\zeta)=\lim _{n \rightarrow \infty} F_{n}(\zeta)=f_{0}(\zeta)+(\zeta-z) f_{0}^{\prime}(z), \quad \zeta \in D_{2}, \quad z=H(\zeta) \in D_{1}
$$

has the complex dilatation $\tilde{\mu}(\zeta)$ given in (3.2).

Finally, $T_{f}=T_{f_{0}}$ implies $f=a f_{0}+b$ for some constants $a, b \in C$, so that $f$ is also univalent in $D_{1}$ and has the quasiconformal extension to $\hat{\mathbf{C}}$, given by (3.1). 


\section{Universal Teichmüller space}

For $n=1,2$, let $B_{n}(D)$ be the Bers' space of analytic functions $\psi$ in a hyperbolic domain $D$, bounded in the norm

$$
\|\psi\|_{n, D}=\|\psi\|_{n}=\sup _{z \in D} \varrho_{D}(z)^{-n}|\psi(z)| .
$$

Let $M(D)$ be the open unit ball in the Banach space $L^{\infty}(D)$ of all complexvalued measurable functions $\mu$ supported in $D$ and bounded in the norm

$$
\|\mu\|_{\infty, D}=\|\mu\|_{\infty}=\underset{z \in D}{\operatorname{ess} \sup }|\mu(z)| .
$$

Let $C, D_{1}, D_{2}$ be as before. For each $\mu \in M\left(D_{2}\right)$ let $f_{\mu}$ be the unique homeomorphic solution of the Beltrami equation

$$
\frac{\partial w}{\partial \bar{z}}=\mu \frac{\partial w}{\partial z}
$$

fixing the points $\{0,1, \infty\}$. Since $\left.\mu\right|_{D_{1}}=0, f_{\mu}$ is conformal in $D_{1}$, so we may define the two mappings

and

$$
\eta_{c}^{(1)}: M\left(D_{2}\right) \rightarrow \mu \rightarrow T_{\mu}=T_{f_{\mu}} \in B_{1}\left(D_{1}\right), \quad T_{f}=f^{\prime \prime} \mid f^{\prime}
$$

$$
\eta_{c}^{(2)}: M\left(D_{2}\right) \rightarrow \mu \rightarrow S_{\mu}=T_{\mu}^{\prime}-\frac{1}{2} T_{\mu}^{2} \in B_{2}\left(D_{1}\right),
$$

the second of which is known (see [6]) as Bers' embedding of the universal Teichmüller space $T\left(D_{2}\right)$ of $D_{2}$ in $B_{2}\left(D_{1}\right)$. The image $T_{1}\left(D_{2}\right)$ of the first mapping $\eta_{c}^{(1)}$ in $B_{1}\left(D_{1}\right)$ may also be considered a representation of $T\left(D_{2}\right)$. Bers proved in [6] the following properties of the embedding $\eta_{c}^{(2)}$ :

Theorem C. Let $C$ be a quasicircle in $\hat{\mathbf{C}}$. The mapping $\eta_{c}^{(2)}: M\left(D_{2}\right) \rightarrow B_{2}\left(D_{1}\right)$ is holomorphic (as a mapping between two complex Banach spaces). Its derivative at the origin is

$$
D \eta_{c}^{(2)}(0): \mu \rightarrow-\frac{6}{\pi} \iint_{D_{2}} \frac{\mu(\zeta)}{(\zeta-z)^{4}} d \xi d \eta, \quad z \in D_{1}, \quad \mu \in L^{\infty}\left(D_{2}\right),
$$

and $D \eta_{c}^{(2)}(0)$ has the right-inverse

$$
\Lambda_{c}^{(2)}: \psi \rightarrow \frac{1}{2}(\zeta-H(\zeta))^{2} \frac{\partial H(\zeta)}{\partial \zeta} \psi(H(\zeta)), \quad \zeta \in D_{2}, \quad \psi \in B_{2}\left(D_{1}\right),
$$

i.e., each $\psi \in B_{2}\left(D_{1}\right)$ satisfies the reproduction formula

$$
\psi(z)=-\frac{3}{\pi} \iint_{D_{z}}(\zeta-H(\zeta))^{2} \frac{\partial H(\zeta)}{\partial \bar{\zeta}} \psi(H(\zeta))(\zeta-z)^{-4} d \xi d \eta, \quad z \in D_{1}
$$

(cf. Lemma 6 in [9]), where $H$ is the quasiconformal reflection at $C$ of Lemma 3. It appears that the embedding $\eta_{c}^{(1)}$ has similar properties: 
Theorem D. Let $C$ be a quasicircle in $\hat{\mathbf{C}}$, and $D_{1}$ and $D_{2}$ its interior and exterior domains, respectively (i.e., $\left.\infty \in \bar{D}_{2}\right)$. The mapping $\eta_{c}^{(1)}: M\left(D_{2}\right) \rightarrow B_{1}\left(D_{1}\right)$ is holomorphic. Its derivative at the origin is the linear mapping

$$
D \eta_{c}^{(1)}(0): \mu \rightarrow-\frac{2}{\pi} \iint_{D_{2}} \frac{\mu(\zeta)}{(\zeta-z)^{3}} d \xi d \eta, \quad z \in D_{1}, \quad \mu \in L^{\infty}\left(D_{2}\right),
$$

and $D \eta_{c}^{(1)}(0)$ has the right inverse

$$
\Lambda_{c}^{(1)}: \psi \rightarrow(\zeta-H(\zeta)) \frac{\partial H(\zeta)}{\partial \zeta} \psi(H(\zeta)), \quad \zeta \in D_{2}, \quad \psi \in B_{1}\left(D_{1}\right),
$$

i.e.,

$$
\begin{gathered}
\psi(z)=-\frac{2}{\pi} \iint_{D_{2}}(\zeta-H(\zeta)) \frac{\partial H(\zeta)}{\partial \zeta} \psi(H(\zeta))(\zeta-z)^{-3} d \zeta d \eta, \\
z \in D_{1}, \quad \psi \in B_{1}\left(D_{1}\right) .
\end{gathered}
$$

Proof. The holomorphicity of $\eta_{c}^{(1)}$ and Formula (4.4) are proven exactly as the corresponding part of Theorem C (see [6] and also [9]). The rest of the theorem follows from Formula (3.2), which means that the mapping

$$
\tilde{\Lambda}_{c}^{(1)}: \psi \rightarrow \mu(\zeta)=\frac{(\zeta-H(\zeta)) \frac{\partial H(\zeta)}{\partial \zeta} \psi(H(\zeta))}{1+(\zeta-H(\zeta)) \frac{\partial H(\zeta)}{\partial \zeta} \psi(H(\zeta))}, \quad \psi \in B_{1}\left(D_{1}\right), \quad \zeta \in D_{2},
$$

is a local cross-section for $\eta_{c}^{(1)}: M\left(D_{2}\right) \rightarrow B_{1}\left(D_{1}\right)$ near the origin, i.e.,

$$
\eta_{c}^{(1)} \circ \tilde{\Lambda}_{c}^{(1)} \psi=\psi \text { for } \psi \in B_{1}\left(D_{1}\right) \text { with }\|\psi\|_{1, D_{1}} \text { small. }
$$

But direct computation easily yields

$$
D \tilde{\Lambda}_{c}^{(1)}(0)=\Lambda_{c}^{(1)},
$$

and (4.6) is derived by applying the chain rule to (4.7). Q.e.d.

\section{References}

[1] Agard, S.: Distortion theorems for quasiconformal mappings. - Ann. Acad. Sci. Fenn. Ser. A I Math. 413, 1968, 1-12.

[2] Ahlfors, L. V.: Quasiconformal reflections. - Acta Math. 109, 1963, 291-301.

[3] Ahrfors, L. V.: Lectures on quasiconformal mappings. - D. Van Nostrand Company, Inc., Princeton, N. J.-Toronto-New York-London, 1966.

[4] Ahlfors, L. V., and G. WellL: A uniqueness theorem for Beltrami equations. - Proc. Amer. Math. Soc. 13, 1962, 975-978.

[5] Astala, K., and F. W. Gehring: Injectivity criteria and the quasidisc. - Complex Variables Theory Appl. 3, 1984, 45-54. 
[6] Bers, L.: A non-standard integral equation with applications to quasiconformal mappings. Acta Math. 116, 1966, 113-134.

[7] Beurling, A., and L. V. Ahlfors: The boundary correspondence under quasiconformal mappings. - Acta Math. 96, 1956, 125-142.

[8] Gehring, F. W.: Univalent functions and the Schwarzian derivative. - Comment. Math. Helv. $52,1977,561-572$.

[9] HaRmelin, R.: Invariant operators and univalent functions. - Trans. Amer. Math. Soc. 272, 1982, 721-731.

[10] Lehto, O., and K. I. Virtanen: Quasiconformal mappings in the plane. - Springer-Verlag, Berlin-Heidelberg-New York, 1975.

[11] Martio, O., and J. Sarvas: Injectivity theorems in plane and space. - Ann. Acad. Sci. Fenn. Ser. A I Math. 4, 1979, 383-401.

Technion

Department of Mathematics

Haifa 32000

Israel

Received 20 Dicember 1985 